
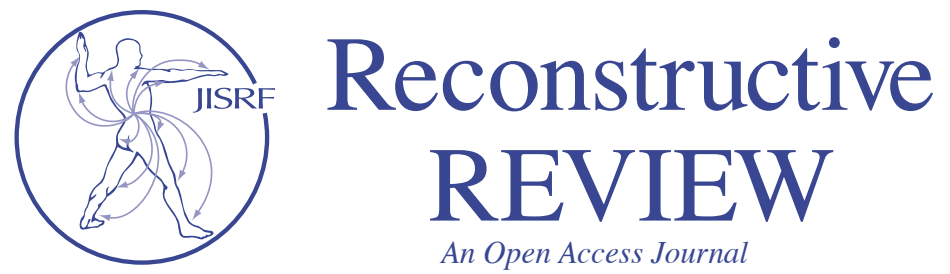

Check for updates

\title{
Overnight or Short Stay Joint Replacements in the Public and Private settings: An Australian Experience
}

\author{
Qurashi, $S^{1} ;$ Bajwa, $S^{2}$; Aktas, $S^{2} ;$ Bestic, $W^{3} ;$ Chinnappa, J4
}

Abstact

Background: In today's post COVID 19 world, many healthcare systems have been pushed past the brink of economic sustainability. With Total Hip (THR) and Knee Replacements (TKR) being some of the biggest ticket items, the need to adopt methods that improve quality of care \& reduce unnecessary costs, is imperative. In this context, we report our experience with a Short Stay / Overnight joint replacement model using an ERAS (Enhanced Recovery After Surgery) Protocol which promotes rapid post-operative recovery and a decreased LOS without an increase in complications or readmission rates.

Method: Retrospective collection of clinical \& demographic data was undertaken for 114 consecutive patients undergoing primary THR or TKR by a single surgeon between 1 January 2018 and 19 March 2020 at 2 hospitals (1 public, 1 private). The data was analyzed for LOS, complications \& readmission rates within 90 days after surgery.

Results: In THR ( $\mathrm{n}=93)$ and TKR $(\mathrm{n}=21)$, mean LOS was1.54 nights (range $0-4$ ). 8 patients were discharged to a rehabilitation facility, the remaining 106 were discharged home. 2 patients were readmitted within 90 days of surgery - one with a periprosthetic fracture and the other for an unrelated respiratory illness.

Conclusion: The implementation of a Short Stay model and associated ERAS protocols in both the public and private hospital settings reduced LOS without a concomitant increase in postoperative complications or readmission rates.

\section{Background}

Joint replacement is the definitive treatment for end stage arthritis. With a $105 \%$ increase in demand for primary TKR \& 73\% for primary THR over the past 10 years [1], an estimated 65,569 patients will require a TKR \& 39,567 a THR annually by 2030 at a cost of AUD 2.38 billion to the healthcare system [2]. This is a major cost to our health system at the best of economic times. Now consider COVID 19 and the resultant AUD \$3.6 billion viral black hole in the Australian economy, the need to be judicious with our healthcare spending is more important than ever [ $\underline{3}]$.

The concept of 'Short stay', 'Overnight', or 'Outpatient' joint replacement surgery has been very talked about in the European and American [4] circles for some years now, but is not an established practice in Australia. Whilst the overseas health system designs are very different, the incentive in Australia has primarily been quicker functional recovery for the patient and less emphasis on the economic motivations. However, our problems on the economic side are no less significant. Elective surgery waitlist blowouts, government funding cuts and now the virus has put a tremendous amount of pressure on our already stretched public system. On the private front, cost of surgery, rehabilitation, and everything that goes with it is astronomical. As per insurance data, the cost of joint replacement in

Keywords: Enhanced recovery after surgery, Knee replacement, Hip replacement, Arthroplasty, Cost-effectiveness

Level of Evidence: IV 
NSW and ACT can be around AUD \$30,000-35,000. Out of this, almost half is attributed to post surgery Length of Stay (LOS) stay and rehabilitation []ㅡ.

LOS is a conventional index of performance, a quality metric [] $]$ at a clinical and also at an economical level. This is due to its objective nature and ease at which it can be measured. In joint replacement surgery, it is a measure of cost as well as efficiency and more recently, found to be associated with better patient satisfaction [7] and reduction in nosocomial complications as well as readmission rates $[\underline{6}, \underline{8}]$.

From an economic standpoint, a major push to reduce LOS stems from healthcare systems employing the Medicare Diagnosis Related Group (DRG) style reimbursement models, whereby the cost of an extended LOS is borne by the hospital and providers [9]. With extended LOS being a significant cause of hospital inefficiencies and expense, many stakeholders are attracted to the idea of cost savings associated with decreased LOS.

On the other hand, concerns about shortening the LOS revolve mainly around a fear of increased complications or readmissions. A landmark study by Meyers et al [10] promoted a week long hospitalization after joint replacement surgery as a norm. This was widely adopted by healthcare systems across the globe in an attempt to prevent post-operative complications. However, in 2021, with newer evidence emerging and advances in perioperative interventions, the above recommendation may be obsolete. There is stronger evidence to show that LOS can be reduced without increasing readmission rates $[\underline{6}, \underline{8}]$.

Additionally, an extended stay in rehabilitation has been shown to be associated with increased costs in addition to increased complications, increased readmission rates and no benefits with regards to patient functional scores and satisfaction rates following hip and knee arthroplasty [11].

In this context, it is imperative to establish healthcare practices that optimize patient care and expedite functional recovery without an increase in morbidity associated with joint replacement surgeries while also reducing the economic burden on healthcare services.

ERAS is a series of evidence based perioperative interventions used in a multimodal, integrated clinical care pathway to achieve accelerated functional recovery $[\underline{12}, \underline{13}]$. It requires the multidisciplinary team including anaesthetists, surgeons, nurses and physiotherapists to adhere to a specifically designed protocol following these principles.

The aim of this investigation is to study if our Short Stay model using a predefined ERAS Protocol achieved reduced LOS (Primary outcome measure) without an increase in complications or hospital readmission rates (secondary outcomes).

\section{Materials and Methods}

After approval from Hunter New England Ethics Committee (Ref: EX202005-03), all patients undergoing elective THR or TKR at St. Luke's Hospital (Private) and Canterbury Hospital (Public) between January 2018 to March 2020 under the care of lead investigator were identified and studied retrospectively. The exclusion criteria were defined as follows: history of malignant hyperthermia, history of allergy to anaesthetic agent, history of substance abuse, impaired cognitive function. A combination of preoperative (patient education and carbohydrate loading), intraoperative (minimizing opioid use, avoiding regional anaesthesia that inhibits mobilization, early analgesia and anti-emetic use) and postoperative (cryocompression, early mobilization, early oral feeding and multimodal analgesia) measures were used in our ERAS protocol. The full details of our ERAS Protocol are provided in Appendix A.

All TKRs (cemented) were performed with standard medial parapatellar approach by the senior author (SQ). All THR were performed using the SuperPATH approach also by the senior author (SQ) with either hybrid or uncemented implants depending on patient factors.

Patient demographics (Age, Gender, BMI, Preoperative Hemoglobin, Postoperative Hemoglobin, LOS and Complications) were retrospectively collected. Patients health status was graded as per ASA Grading system from Grade I (Healthy) to Grade V (Moribund) [14]. All patients had planned follow ups at 2,6 weeks postoperatively for Xray and clinical evaluation (Wound status, Range of Motion and functional status).

\section{Outcome measures}

The primary outcome measure was LOS postoperatively, defined as the number of nights in the hospital from the date of surgery. The secondary outcome measures were complications (wound complications, falls during post-operative period, periprosthetic fracture, neurovascular injury, infection, dislocation, venous thromboembolism, any infections, or any other complication associated with surgery and readmission into the hospital after discharge for any cause within 90 days of surgery.

\section{Statistical Analysis}

The sample size was determined with convenience sampling using 'Sample size calculator- The survey system'. Using 5 cases per month over an enrolment period of 25 months, 112 patients were required for a $95 \%$ Confidence Interval with a 5\% margin of error. Demographics and baseline characters were evaluated using the geometric mean. 
The primary and secondary outcome measures are presented as medians and ranges. The continuous variables in secondary outcome measures were assessed for normality. Statistical analysis of the data was performed using Graph Pad Prism for MacOSX, GraphPad Software, California, USA.

\section{Results}

We recruited 114 consecutive THR and TKR's from 1st January 2018 to 18th March 2020. 72 patients underwent surgery in the public system at Canterbury Hospital (54 THR and 18 TKR) while 42 patients were at St. Luke's Private Hospital (39 THR and 3 TKR). This was a total of 93 patients undergoing THR and 21 patients undergoing TKRs. No patients met the exclusion criterion and hence every patient in this time frame was included in the study. Table 1 represents the patient demographics.

Table 1-Patient Demographics

\begin{tabular}{|l|c|c|c|}
\hline & THR (Range) & TKR (Range) & Total (114) \\
\hline Age (Years) & $62.52(33-82)$ & $71.39(56-82)$ & 64.15 \\
\hline Sex (Female) & $47 / 93$ & $12 / 21$ & $59 / 114$ \\
\hline BMI & $28.19(18.2-45.9)$ & $30.72(20.4-39.9)$ & 29.46 \\
\hline \multicolumn{4}{|c|}{ ASA Grading } \\
\hline Grade I & 13 & 2 & 15 \\
\hline Grade II & 57 & 14 & 71 \\
\hline Grade III & 22 & 5 & 27 \\
\hline Grade IV & 1 & 0 & 1 \\
\hline
\end{tabular}

\section{Length of Stay}

The mean LOS for patients undergoing THR was 1.45 nights (Range: same day discharge - 4) and in patients undergoing TKR was 1.90 nights (Range: 1 - 4). The combined mean LOS was 1.54 nights. (Table 2). 87 of all THR patients and 19 of TKRs were discharged home to continue physiotherapy autonomously. The remaining patients opted to pursue physiotherapy at a rehabilitation center (Table 2 ). All of the patients that were discharged to a rehabilitation unit had indicated their intention to do so preoperatively instead of a deemed requirement post surgery.

Most patients were ready for discharge after 1 night post operatively (as per our discharge criteria). In patients that stayed longer, the reason for delayed discharge were: postoperative vomiting $(\mathrm{n}=1)$, wound ooze requiring application of PICO incisional suction dressing $(n=1)$, urinary retention requiring catheterization overnight $(n=1)$, fall during hospitalisation ( $\mathrm{n}=1$ - this patient remained asymptomatic however imaging was performed to exclude pathology and more physiotherapy instructional sessions on maneuvering walking aids were provided delaying planned discharge) and delay in organising logistics for interstate / regional travel or awaiting a rehab bed $(n=4)$. In the last group, the reasons were primarily social or logistical even though they had been cleared by physiotherapy/ allied health as well as medical staff. Regardless of the cause of delay, all patients were discharged within 4 nights of surgery. It is also worth noting that if patients felt that they were not ready (social reasons), they were allowed to stay even if they had been cleared for safe discharge.

\section{Complications}

Two patients had to be readmitted unexpectedly after presenting to the Emergency Department (ED) - one had a periprosthetic fracture and the other presented with an unrelated respiratory viral illness (table 2).

Table 2- Complications

\begin{tabular}{|c|c|c|c|}
\hline & THR (Range) & TKR (Range) & Total (114) \\
\hline $\begin{array}{l}\text { Length of Stay } \\
\text { (Nights) }\end{array}$ & $\begin{array}{c}1.45 \\
\text { (Same day } \\
\text { discharge- } 4)\end{array}$ & $1.90(1-4)$ & 1.54 \\
\hline \multicolumn{4}{|c|}{ Discharge Status } \\
\hline Home & $87 / 93$ & $19 / 21$ & $106 / 114$ \\
\hline $\begin{array}{l}\text { Rehabilitation } \\
\text { Center }\end{array}$ & $6 / 93$ & $2 / 21$ & $8 / 114$ \\
\hline $\begin{array}{l}\text { Preoperative } \\
\text { Hemoglobin }\end{array}$ & $140.5(107-178)$ & $138(111-158)$ & 139.3 \\
\hline $\begin{array}{l}\text { Postoperative } \\
\text { Hemoglobin }\end{array}$ & $\begin{array}{c}114.3(86-142) \\
(1 \text { post op } \\
\text { Inj Ferratin } \\
1000 \mathrm{mg})\end{array}$ & $115.6(97-143)$ & 114.9 \\
\hline $\begin{array}{l}\text { Readmissions } \\
\text { (within } 30 \text { days } \\
\text { of surgery) }\end{array}$ & $\begin{array}{c}1 \\
\text { (Peri-prosthetic } \\
\text { Fracture) }\end{array}$ & $\begin{array}{c}1 \\
\text { (Viral } \\
\text { infection) }\end{array}$ & 2 \\
\hline \multicolumn{4}{|c|}{ Complications } \\
\hline $\begin{array}{l}\text { Fall during } \\
\text { Hospitalisation }\end{array}$ & $\begin{array}{c}1 \\
\text { (No } \\
\text { complications) }\end{array}$ & & 1 \\
\hline \multicolumn{4}{|c|}{ Infections } \\
\hline $\begin{array}{l}\text { Stitch Abscess/ } \\
\text { Wound } \\
\text { Complications }\end{array}$ & & $\begin{array}{c}1 \\
\text { (Stitch } \\
\text { Abscess) }\end{array}$ & 1 \\
\hline
\end{tabular}

The patient with the periprosthetic fracture had been discharged essentially pain free on day one post surgery and independently mobile on crutches. Towards late afternoon of day 3 she contacted the surgeon's rooms to advise that she started getting pain that afternoon after being asymptomatic earlier. This had become severe so she was ad- 
vised to present to the hospital and a CT scan showed an undisplaced femoral calcar fracture. She was treated nonoperatively with partial weight bearing for 4 weeks, with sequential radiographs demonstrating a stable implant. She was discharged from the hospital a few days later. At 6 week follow-up, she was independently mobile with no walking aids, no pain and no radiological abnormalities.

One patient had an unplanned visit to the outpatient clinic with a minor stitch abscess which did not require an admission after settling with oral antibiotic treatment.

No patient required a blood transfusion and one asymptomatic patient required a ferritin injection postoperatively for a low haemoglobin $(\mathrm{Hb})$. The mean preoperative $\mathrm{Hb}$ was $140.5 \mathrm{gm} / \mathrm{L}$ (Range: 107- 178) in THR group and $138 \mathrm{gm} / \mathrm{L}$ (range: 111- 158) in the TKR group with a combined mean of $139.3 \mathrm{gm} / \mathrm{L}$. The mean postoperative $\mathrm{Hb}$ was $114.3 \mathrm{gm} / \mathrm{L}$ (range: $86-142$ ) in the THR group and $115.6 \mathrm{gm} / \mathrm{L}$ (range: 97- 143) in TKR group with a combined mean of $114.9 \mathrm{gm} / \mathrm{L}$.

\section{Discussion}

A number of studies [15-17] promoted Short Stay programs / ERAS protocols to be effective in reducing the LOS without increasing the morbidity or hospital expenditure and thereby giving an accelerated recovery in the perioperative period. Our study confirms that Short Stay / ERAS can be implemented in both public and private hospitals in the Australian healthcare landscape and achieve LOS much lower than what is quoted in the literature as being associated with such models. A large number of our patients were deemed suitable for discharge within 1-2 nights postoperatively according to our discharge criteria.

However, for successful application of such a protocol, a truly multidisciplinary collaborative involvement is essential. Efficient implementation of the protocol at every level may be difficult especially if staff are used to long standing conventional practices with other surgical teams in the same institution

After preoperative patient education and hospital admission, the type of anaesthetic recipe played a vital role. Systemic evaluation preoperatively, intraoperative tranexamic acid, controlled hemostasis and adrenaline in LIA helped minimise blood loss. Emphasis on reduction of PONV (Postoperative Nausea \& Vomiting) \& POCD (Postoperative Cognitive Dysfunction) compared to other studies [18] due to our use of short acting drugs such as fentanyl, midazolam, vercuronium and propofol helped quicken anaesthetic recovery. Generous use of LIA reduced immediate postoperative pain and minimised the need for long act- ing opioids. These interventions along with the absence of surgical drains [19] and urinary catheters [마] facilitated early mobilisation, as did avoidance of routine spinal analgesia [21] and nerve blocks with associated quadriceps weakness [22]. The choice of postoperative analgesic regimen facilitated patient capability during physiotherapy. From the time the patient woke up, oral Oxycodone and/or SR Topentadol were used regularly as well as rescue medicines if needed. Prompt oral intake were encouraged with minimum use of IV fluids.

With ERAS protocols, ward practices including nursing care and physiotherapy / allied staff and administrative practices were all adjusted. Multiple inefficient administrative practices also contribute to delay in discharge [23] One such administrative practice was a surprising find, whereby there was a reluctance of private hospitals to engage in the 'short stay' model. This was constantly blamed on their individual contracts with the health funds, even citing financial penalties (from health funds) or reduced 'case payments' for a discharge earlier than their stipulated number of nights, four nights being the one most often quoted. This reluctance drained its way down from management levels and into nursing and allied staff practices making it obstructive to short stay plans in some instances. The basis of these practices originated from administrators and insurers being focused on reducing implant costs and other smaller savings rather than the major expenses such as LOS and rehabilitation costs for fear of complications and readmissions. We know now that the data used to justify this stance is not applicable in 2021. ERAS has shown to effectively reduce hospital related costs, perioperative morbidity and improve patient satisfaction in the literature [24] and consistent with our experience. Moreover, we also know that blanket inpatient rehabilitation for all, another practice that is sold to patients as being 'a must for all' has been shown to achieve the contrary outcome, with increased readmission rates, costs and complications and no improvements in patient functional scores or satisfaction [11].

We aimed to demonstrate that an accelerated pathway for arthroplasty can work well with the involvement \& training of the whole team. With Short Stay, LOS was chosen as the primary measuring outcome as it is the most important reflective index of hospital costs and an overall measure of team efficiency. LOS is a parameter of concern to each physician but also is a tool to reduce the overall burden of healthcare costs to governments around the globe.

Compared to large scale comparative studies in the recent past (Traditional vs Short Stay / ERAS principles) in Arthroplasty [25], our complication rates are far lower in 
mortality ( $0.1 \%$ vs None in our study), with lower LOS and blood transfusion ( $9.8 \%$ vs None). Our findings are similar to another Australian study investigating their use of local anaesthetic infiltration to allow a rapid recovery protocol following hip and knee replacements in the private sector with significant associated cost savings [15]. Our readmission rates of THR (1\%) and TKR (4\%) are slightly lower (3.9\% and 6\% respectively) with similar mean LOS.

The limitations of our study are our small sample size and retrospective nature of the study. It was also difficult to evaluate the impact of our surgical technique (SuperPATH in Hip Arthroplasty) on ERAS or its individual components. We did not undertake any analysis of the patient satisfaction scores or a health cost analysis which would be components to study in future prospective trials to better establish the effects of the Short Stay protocol \& its individual components.

\section{Conclusion}

With the partnership of motivated staff and educated patients, Short Stay / ERAS protocols can be implemented in patients undergoing joint replacements in Australian public and private hospitals alike to improve the outcome parameters without any adverse effect on complication rates or readmissions.

\section{References}

1. Ackerman I, Bohensky M, de Steiger R, Brand C, Eskelinen A, Fenstad A et al. Substantial rise in the lifetime risk of primary total knee replacement surgery for osteoarthritis from 2003 to 2013: an international, population-level analysis. Osteoarthritis and Cartilage. 2017;25(4):455-461.

2. Ackerman I, Bohensky M, Zomer E, Tacey M, Gorelik A, Brand C et al. The projected burden of primary total knee and hip replacement for osteoarthritis in Australia to the year 2030. BMC Musculoskeletal Disorders. 2019;20(1).

3. Coronavirus set to punch $\$ 360 \mathrm{~b}$ hole in Budget. Here's how you might pay for it [Internet]. 2020 [cited 2020 May 12]. Available from: https://www.abc.net.au/ news/2020-05-11/economic-cure-post-coronavirus-is-tax-reform/12227760

4. Hoffmann JD, Kusnezov NA, Dunn JC, Zarkadis NJ, Goodman GP, Berger RA. The shift to same-day outpatient joint arthroplasty: A systematic review. J Arthroplasty. 2018;33(4):1265-74.

5. RACS Surgical Practice Variation Report - Orthopaedic procedures 2016,

6. Sarpong NO, Boddapati V, Herndon CL, Shah RP, Cooper HJ, Geller JA. Trends in length of stay and 30-day complications after total knee arthroplasty: An analysis from 2006 to 2016. J Arthroplasty. 2019;34(8):1575-80.

7. Husted H, Holm G, Jacobsen S. Predictors of length of stay and patient satisfaction after hip and knee replacement surgery: fast-track experience in 712 patients. Acta Orthop. 2008;79(2):168-73.

8. Sutton JC 3rd, Antoniou J, Epure LM, Huk OL, Zukor DJ, Bergeron SG. Hospital discharge within 2 days following total hip or knee arthroplasty does not increase major-complication and readmission rates. J Bone Joint Surg Am. 2016;98(17):1419-28.

9. Auyong DB, Allen CJ, Pahang JA, Clabeaux JJ, MacDonald KM, Hanson NA. Reduced length of hospitalization in primary total knee arthroplasty patients using an updated enhanced recovery after orthopedic surgery (ERAS) pathway. J Arthroplasty. 2015;30(10):1705-9.

10. Meyers S, Reuben J, Cox D, Watson M. Inpatient cost of primary total joint arthroplasty. The Journal of Arthroplasty. 1996;11(3):281-285.

11. Onggo JR, Onggo JD, De Steiger R, Hau R. The efficacy and safety of inpatient rehabilitation compared with home discharge after hip or knee arthroplasty: A meta- analysis and systematic review. J Arthroplasty. 2019;34(8):1823-30.

12. Ibrahim M, Khan M, Nizam I, Haddad F. Peri-operative interventions producing better functional outcomes and enhanced recovery following total hip and knee arthroplasty: an evidence-based review. BMC Medicine. 2013;11(1).

13. Miller T, Thacker J, White W, Mantyh C, Migaly J, Jin J et al. Reduced Length of Hospital Stay in Colorectal Surgery after Implementation of an Enhanced Recovery Protocol. Anesthesia \& Analgesia. 2014;118(5):1052-1061.

14. Mayhew D, Mendonca V, Murthy B. A review of ASA physical status - historical perspectives and modern developments. Anaesthesia. 2019;74(3):373-379.

15. Nassar I, Fahey J, Mitchell D. Rapid recovery following hip and knee arthroplasty using local infiltration analgesia: length of stay, rehabilitation protocol and cost savings. ANZ Journal of Surgery. 2020;90(3):355-9.

16. Christelis N, Wallace S, Sage C, Babitu U, Liew S, Dugal J et al. An enhanced recovery after surgery program for hip and knee arthroplasty. Medical Journal of Australia. 2015;202(7):363-368.

17. Ljungqvist O, Scott M, Fearon K. Enhanced Recovery After Surgery. JAMA Surgery. 2017;152(3):292

18. Homburger J, Meiler S. Anesthesia drugs, immunity, and long-term outcome. Current Opinion in Anaesthesiology. 2006;19(4):423-428.

19. Quinn M, Bowe A, Galvin R, Dawson P, O'Byrne J. The use of postoperative suction drainage in total knee arthroplasty: a systematic review. International Orthopaedics. 2014;39(4):653-658.

20. Bjerregaard L, Hornum U, Troldborg C, Bogoe S, Bagi P, Kehlet H. Postoperative Urinary Catheterization Thresholds of 500 versus $800 \mathrm{ml}$ after Fast-track Total Hip and Knee Arthroplasty. Anesthesiology. 2016;124(6):1256-1264.

21. Kehlet H, Aasvang E. Regional or general anesthesia for fast-track hip and knee replacement - what is the evidence?. F1000Research. 2015;4:1449.

22. Chaumeron A, Audy D, Drolet P, Lavigne M, Vendittoli P. Periarticular Injection in Knee Arthroplasty Improves Quadriceps Function. Clinical Orthopaedics and Related Research®. 2013;471(7):2284-2295.

23. Maessen J, Dejong C, Hausel J, Nygren J, Lassen K, Andersen J et al. A protocol is not enough to implement an enhanced recovery programme for colorectal resection. British Journal of Surgery. 2007;94(2):224-231.

24. Husted H, Lunn T, Troelsen A, Gaarn-Larsen L, Kristensen B, Kehlet H. Why still in hospital after fast-track hip and knee arthroplasty?. Acta Orthopaedica. 2011;82(6):679-684.

25. Khan S, Malviya A, Muller S, Carluke I, Partington P, Emmerson K et al. Reduced short-term complications and mortality following Enhanced Recovery primary hip and knee arthroplasty: results from 6,000 consecutive procedures. Acta Orthopaedica. 2013;85(1):26-31.

\section{Appendix A: ERAS Protocol}

Preoperative:

Preoperative Joint Replacement education/counselling in the clinics by the Chief operating surgeon to the patient and their immediate family / partner.

Preoperative assessment done by the anaesthetist in charge to discuss the anaesthetic walk through for the procedure.

Premedication-Oral analgesic was given preoperatively. Avoid sedatives (Benzodiazepines, Neuroleptics or Opioids given preoperatively)

Preoperative fasting of 6 hours with clear fluids allowed till 2 hours preoperatively. Preoperative carbohydrate loading.

Preoperative warming of patient with warmers and blankets was implemented from ward to preoperative anaesthetic bay.

Intraoperative:

General anaesthesia as a standard approach. No nerve blocks that impair motor function and early mobilisation.

Minimum use of opioids.

Drugs used:

\section{Midazolam}

Propofol for induction then TCI

Fentanyl approx. $300 \mathrm{mcg}$ for entire case $(50 \mathrm{mcg}$ intubation, $100 \mathrm{mcg}$ pre incision, $100 \mathrm{mcg}$ during, $50 \mathrm{mcg}$ prior to wake up)

Vecuronium $20 \mathrm{mg}$ intubation and $10 \mathrm{mg}$ bolus as required during case

Paracetamol 1g then chart QID for 5 days post operative

Parecoxib 40mg then chart NSAID for 3-5 days post operative

Tramadol 200mg pre incision

Ketamine 50mg pre incision

Dexamethasone $8 \mathrm{mg}$

Ondansetron $8 \mathrm{mg}$ and chart $\mathrm{PRN}$ post operative

If other indicators for PONV then add metoclopramide $20 \mathrm{mg}$ and cyclizine $50 \mathrm{mg}$

Sugammadex $200 \mathrm{mg}$ 
- $\quad 1 \mathrm{~g}$ Tranexamic Acid IV 15 minutes before incision followed by 2 doses post-operatively at 8 and 16 hours post-operation

- $\quad$ Cefazolin $2 \mathrm{~g}$ post intubation then chart $3 \mathrm{x}$ doses $\mathrm{q} 8 \mathrm{~h}$ post operative

- IVF 1L intraop with second litre started before end of case. $3 \times 1 \mathrm{~L}$ post op (q5h, q8h, q12h)

- $\quad$ Continued intraoperative warming by warmed IV infusion \& air warming.

- $\quad$ No indwelling catheters and no drains during or after surgery.

- $\quad$ Application of intermittent pneumatic compression device (IPCD) to the leg opposite to surgical side.

- $\quad$ Local Infiltrative Analgesia (LIA) of Ropivacaine $(0.2 \%)+$ adrenaline after arthrotomy, during the procedure and closure.

Postoperative:

- $\quad$ No PCA/ No IDC (tethers patient to bed and increases risk of infection)

- $\quad$ Discontinue IV fluids after surgery when the vital parameters are stable. Start oral feeds early.

- $\quad$ Antiemetic prophylaxis.

- $\quad$ Ice packs for 30 minutes every 2 hours as cryo-compression.

- DVT Prophylaxis with intermittent pneumatic compression devices (IPCD), compression socks and enoxaparin (LMWH) SC injections for 10 days post-operation.

- Multimodal analgesia: Regular Paracetamol + NSAID, Tapentadol SR 50mg BD for 3-4 days and Tapentadol IR 50mg q4h PRN. Oxycodone for PACU $1 \mathrm{mg}$ q5mins max 5-8mg depending on patient

- Patient goes home with post op pain sheet and scripts

- Early postoperative (recovery room) oral carbohydrate supplementation

- Patients are reviewed on ward end of list to ensure they have mobilized

- Physiotherapy initiated on day of surgery. Patient were made to walk with a walking frame on the day of surgery. Mobilisation Protocol: Mobilisation within 24 hours

- $\quad$ POD 0: Assisted walking, bed to chair transfer.

- POD 1: Independent walking (with gutter crutches), stair climbing and independent transfers.

- $\quad$ Early hospital discharge ( $<5$ days). Discharge criteria was identified to be when the patient mobilized independently, was able to climb stairs and do an independent bed to chair transfer, provided medical indices were normal and patient comfortable.
SUBMISSION HISTORY

Submitted: June 8, 2021

Reviewed: August 21, 2021

Accepted: September 16, 2021

Published: October 12, 2021

AUTHOR AFFILIATIONS

1 Sol Qurashi, MD; The Hip and Knee Clinic, Harbourcity Orthopaedics, Sydney, NSW, Australia; Nepean Hospital, Penrith, NSW, Australia; Canterbury Hospital, Campsie, NSW, Australia

2 Supreet Bajwa, MD; Sam Aktas, MN; The Hip and Knee Clinic, Harbourcity Orthopaedics, Sydney, NSW, Australia

3 William Bestic, MD; Royal North Shore Hospital, St. Leonard's, NSW, Australia

4 Jason Chinnappa, MD; Sydney Orthopaedic And Sports Injury Service, Sydney, NSW, Australia

(Direct inquires to Sol Qurashi, info@harbourcityorthopaedics.com.au)

AUTHOR DISCLOSURES

- The authors declare that there is no conflict of interest in connection with this submitted article.

\section{COPYRIGHT \& OPEN ACCESS}

(C) 2021 Qurashi, Bajwa, Aktas, Bestic, Chinnappa. All rights reserved. Authors retain copyright and grant the journal right of first publication with the work. Reconstructive Review is an open access publication and follows the Creative Commons Attribution-NonCommercial CC BY-NC. This license allows anyone to download works, build upon the material, and share them with others for non-commercial purposes as long as they credit the senior author, Reconstructive Review, and the Joint Implant Surgery \& Research Foundation (JISRF). An example credit would be: "Courtesy of (senior author's name), Reconstructive Review, JISRF, Chagrin Falls, Ohio". 